\title{
Bilateral Tibia Stress Fractures: Case Report and Review of the Literature
}

Nabil Ebraheim MD, Jiayong Liu PhD, Robert Steiner MD, Kyle Andrews MD*, Lucas Mckean BS and Jacob Stirton MD

Department of Orthopaedics, The University of Toledo Medical Center, 1125 Hospital Dr. Toledo, Ohio 43614, USA

*Corresponding author: Kyle Andrews, MD, Department of Orthopaedics, The University of Toledo Medical Center, 1125 Hospital Dr., Toledo, Ohio 43614, USA, Tel: +419-383-4000; E-mail: Kyle.Andrews3@UToledo.Edu

Rec date: June 02, 2017; Acc date: August 07, 2017; Pub date: August 09, 2017

Copyright: $\odot 2017$ Ebraheim N, et al. This is an open-access article distributed under the terms of the creative commons attribution license, which permits unrestricted use, distribution, and reproduction in any medium, provided the original author and source are credited.

\section{Abstract}

Background: Tibia stress fractures are microscopic fractures of the tibia that are often the result of repetitive microtrauma as commonly seen in military recruits and intense athletics. Tibia stress fractures are more commonly unilateral and are usually in the diaphyseal portion of the tibia. We describe a case of bilateral medial proximal tibia occult fractures along the epiphyseal scar following blunt trauma.

Case description: A 41-year-old bipolar male presented to the clinic one-week following a pedestrian versus motor vehicle accident. He subsequently developed low back pain and bilateral knee pain. The diagnosis of bilateral proximal tibia fractures was made using repeat plain X-ray films two-weeks after initial presentation. Operative treatment with bilateral medial tibia internal fixation was elected and the patient was discharged full weight-bearing.

Literature review: There have been two case reports which explored the treatment options for bilateral proximal tibia fractures. Neither case elected to treat with internal fixation.

Clinical relevance: Because the incidence of bilateral tibia stress fractures is low and operative treatment with bilateral internal fixation has not been reported, our case examines the viability of operatively treating bilateral tibia stress fractures with internal fixation. Our case demonstrates the successful treatment of bilateral medial proximal tibia occult fractures with internal fixation.

Keywords: Tibia Fractures; Microtrauma; Blunt trauma; Bilateral internal fixation; Injury

\section{Introduction}

Tibia stress fractures occur in bone that undergoes repetitive load, leading to microscopic fractures that do not completely heal. These injuries are commonly seen in military recruits and athletes as a result of cyclical load with incomplete rest. During intense exercise, bone resorption exceeds bone formation although the exact mechanism remains unclear [1]. The patient usually presents with insidious onset of pain that worsens with increased activity. Over time with repeated injury, the pain will not remit with rest and the athlete will have constant pain in the location of injury. The typical location for tibia stress fractures is diaphyseal which is considered low-risk [1]. Low-risk stress fractures have a favorable prognosis when treated with activity restriction. However, high-risk stress fractures have a higher propensity to develop delayed union or nonunion, especially if the diagnosis is delayed [2]. Only one case report was found describing bilateral medial proximal tibia stress fractures at the epiphyseal scar, associated with athletic activity. In this case study, we present a case of bilateral medial proximal tibia occult fractures along the epiphyseal scar following blunt trauma.

\section{Case Report}

The patient is a 41-year-old male with a history of bipolar disorder and chronic back pain who was seen in the orthopaedic clinic following a pedestrian versus motor vehicle accident one-week prior with a chief complaint of back pain and new-onset bilateral knee pain. The patient was unsure of the exact mechanism of how he was hit, but describes being hit in the leg by a car traveling approximately 15 miles per hour. The patient is a one pack-per-day smoker for ten years and was being treated with dilantin (phenytoin) for bipolar disorder. $\mathrm{He}$ initially presented to an outside hospital where plain film X-rays were obtained of his knees and back, which the patient reported as "normal."

The patient had a history of a previous motor vehicle accident about 15 years ago leading to his chronic back pain. The patient had an MRI from approximately 1 year ago showing a mild circumferential disc bulge at L4-5 with mild neural foraminal narrowing and an L5-S1 disc protrusion without spinal canal stenosis or foraminal narrowing. At his initial exam, he was found to have 9/10 pain in his lumbar spine and bilateral anterior knees. A complete spine exam was performed showing decreased lumbar motion and paraspinal tenderness. No radicular signs or symptoms were found and his motor strength was $5 / 5$ in all extremities. A complete knee exam was done showing tenderness over the anterior and medial knees, small superficial abrasions to bilateral knees, and no evidence of knee effusions. His motion and stability were within normal limits. X-rays were obtained in our clinic showing minimal medial joint space narrowing and spurring of bilateral knees (Figure 1).

His lumbar spine films were also benign. The initial plan was conservative management with bilateral knee compression sleeves and an MRI of his lumbar spine with follow-up set after completion of the MRI over the next week. 


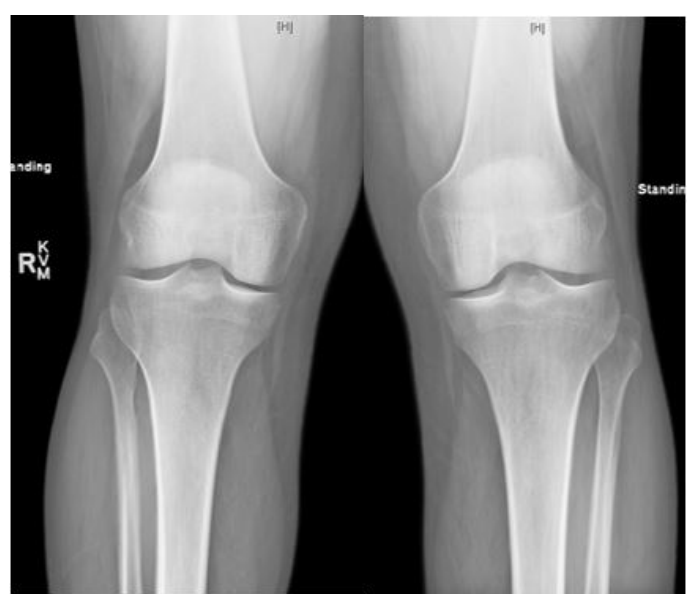

Figure 1: Left knee and right knee anterior-posterior (AP) standing radiographs at initial presentation.

The patient had called the clinic prior to his lumbar spine MRI describing increased knee pain and subjective instability. Bilateral knee MRIs were ordered to be done at the time of his previously ordered lumbar spine MRI. The patient had his MRIs done approximately two weeks later and returned to clinic the next day. His follow-up exam showed continued lumbar paraspinal tenderness as before. His knee exam demonstrated tenderness over the medial joint line of bilateral knees, no mechanical symptoms and no effusion. The knees remained stable with normal range of motion and strength. His MRI of the lumbar spine was reviewed which showed no change to his previous L4-5 and L5-S1 disc bulges with minimal canal or foraminal stenosis. Repeat knee X-rays showed bilateral medial tibia metaphyseal occult fractures over the epiphyseal scar with sclerotic densities along the fracture lines (Figure 2).

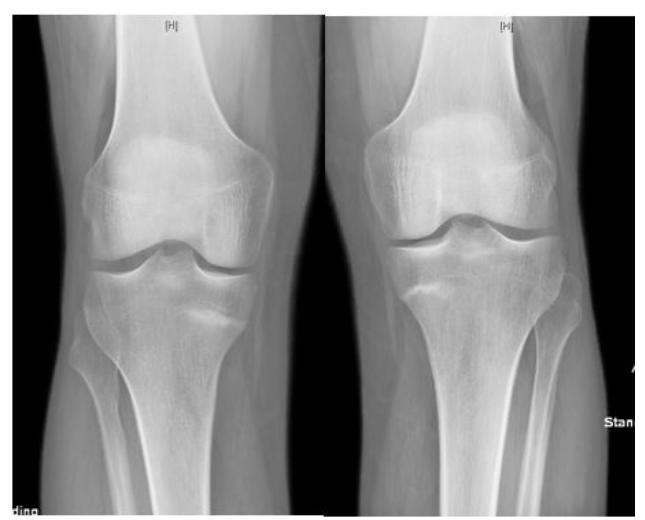

Figure 2: Bilateral knee AP standing radiographs taken 2 weeks later. Sclerotic densities present along medial epiphyseal scars, consistent with occult fractures.

His knee MRIs showed occult fracture lines along the medial tibia metaphysis bilaterally. The fractures remained extra-articular and extended approximately $70 \%$ to $80 \%$ across the tibia metaphysis in the sagittal plane and $40 \%$ to $60 \%$ of the metaphysis in the coronal planes (Figure 3).

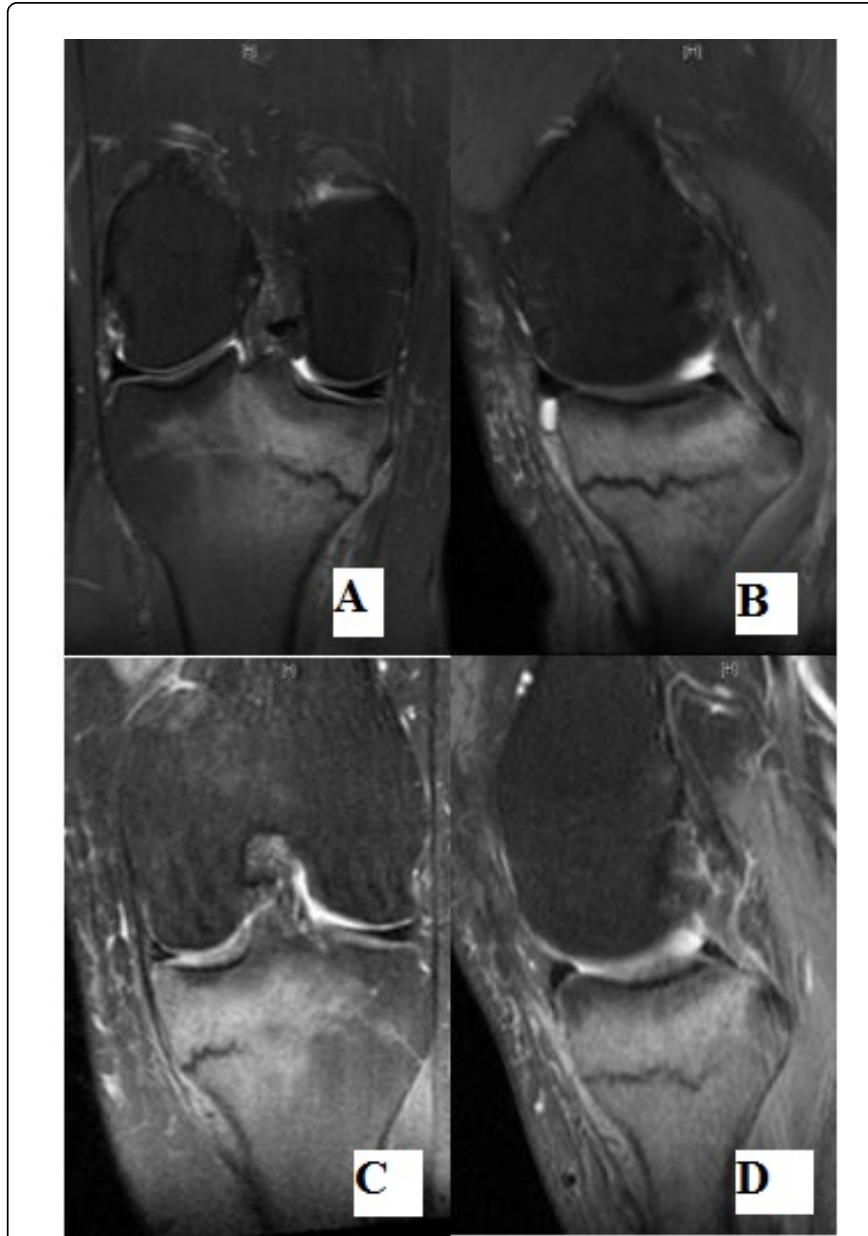

Figure 3: MRI Right knee: Coronal (A), Sagittal (B). Fracture line evident along medial epiphyseal scar. MRI Left knee: Coronal (C), Sagittal (D), showing similar fracture pattern along epiphyseal scar.

We had a long discussion with the patient regarding treatment options, including conservative versus operative management. The patient wanted to return to an active lifestyle as quickly as possible and wished to proceed with operative fixation of these fractures. The patient was set up for surgery and a full metabolic work-up was completed. His BMP, magnesium, calcium, TSH, phosphorus, CBC, ESR, and PTH were all within normal limits. He did have decreased vitamin D 25-OH (20.5 (ref 30.0- 80.0)), elevated C-reactive protein (8.3 (ref $0.0-7.0)$ ), and elevated bone specific alkaline phosphatase (33.7 (ref 6.5-20.1)).

The patient was taken to surgery five days later where a minimallyinvasive two-incision approach was performed for internal fixation of bilateral medial proximal tibia occult fractures (Figure 4).

The fractures were maintained in anatomic alignment and bilateral medial proximal tibia locking plates (Synthes) were used for fixation. The patient was discharged full weight-bearing. Follow-up showed adequate fracture healing with an uneventful recovery. 


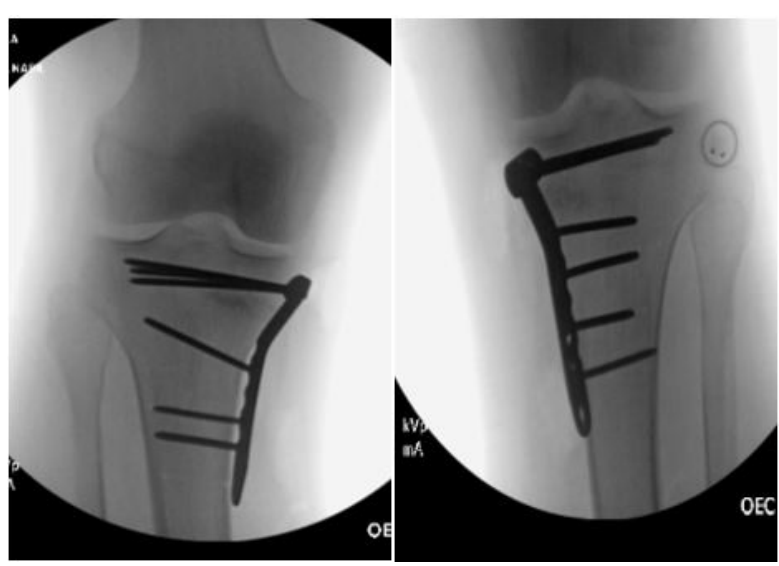

Figure 4: Intraoperative AP images of bilateral knees after fixation using medial T-locking plates.

\section{Discussion}

Tibia stress fractures are the result of repeated microtrauma to the tibia without sufficient rest for healing and continued strain on the bone [3]. These fractures are common among athletes in sports that expose the athlete to repeated stresses such as cross-country and gymnastics [4]. Female athletes tend to suffer stress fractures more often with a relative risk reported 1.5 to 3.5 compared to males. Our patient's presentation is unique in that he presented after acute trauma, likely without a rapid increase in physical activity or repeated lowstress injury. While our patient did have risk factors for bone pathology (smoking and vitamin D deficiency), the timing of injury is unique.

Additionally, stress fractures are usually unilateral, however our patient presented with bilateral stress fractures. One study, published in 2012, showed the incidence of bilateral injury in someone presenting with a stress fracture was $16 \%$ [5]. This result may suggest that while our patient is in the minority, it is not uncommon for bilateral pathology to be present. It is our belief, that physicians should have a high index of suspicion for bilateral injury in patients presenting with a stress fracture and not assume only unilateral pathology. Diagnosing bilateral injury could help guide treatment decisions as well as inform the patient of the need for lifestyle modifications and continued rest to prevent further injury.

The typical management of low-risk tibia fractures is cessation of the inciting activity with resolution within 4 weeks to 8 weeks. Low- risk fractures are tibia shaft fractures and fractures that don't occur in the anterior cortex or the medial malleolus [6]. The anterior cortex is relatively less vascularized and delayed healing is often noted in these fractures. In our patient, the stress fractures were in the proximal tibia, so risk-assessment was difficult. However, it is likely his risk-factors for poor fracture healing and the bilateral nature of his injury pointed towards a lower chance of resolution with conservative measures.

In addition, our patient presented with medial epiphyseal fracture lines noted on MRI. With this picture, a grade IV tibia fracture is likely with expected healing time exceeding 16 weeks [7]. This patient was eager to return to physical activity and failed several weeks of conservative management, including compression sleeve, prior to MRI. Due to the bilateral nature of his injury, several risk factors for poor healing, and failure of conservative management the decision was made to use internal fixation with bilateral Synthes locking plates and the patient was discharged full weight-bearing.

\section{Conclusion}

In the case presented, bilateral proximal medial epiphyseal tibia stress fractures were noted on MRI and were subsequently fixed with proximal tibia locking plate. Prior to surgery, the patient had failed conservative measures with compression sleeve and rest and was emphasizing desire to return to his active lifestyle. Surgical intervention was chosen due to failure of conservative measures and the presence of risk factors associated with poor fracture healing. Long-term results are unknown at this time.

\section{References}

1. Boden BP, Osbahr DC, Jimenez C (2001) Low-risk stress fractures. Am J Sports Med 29: 100-111.

2. Boden BP, Osbahr DC (2000) High-risk stress fractures: Evaluation and treatment. J Am Acad Orthop Surg 8: 344-353.

3. Siparsky PN, Kanaan MG, Garrett WE (2013) Bilateral proximal tibial stress fractures at the epiphyseal scar. The Duke Orthop J 3: 71-73.

4. Changstrom BG, Brou L, Khodaee M, Braund C, Comstock RD (2015) Epidemiology of stress fracture injuries among US high school athletes, 2005-2006 through 2012-2013. Am J Sports Med 43: 26-33.

5. McCormick F, Nwachukwu BU, Provencher MT (2012) Stress fractures in runners. Clin Sports Med 31: 291-306.

6. Chen WM, Huang CK, Chen TH, Chiang CC, Lo WH (2004) Bilateral proximal tibial stress fractures in osteoarthritic knee treated with simultaneous corrective osteotomy and internal fixation. J Chin Med Assoc 67: 48-50.

7. Shindle MK, Endo Y, Warren RF, Lane JM, Helfet DL, et al. (2012) Stress fractures about the tibia, foot and ankle. J Am Acad Orthop Surg 20: $167-176$. 